\title{
The role of the random magnetic fields in the ISM: HVC numerical simulations
}

\author{
Alfredo Santillán ${ }^{1} \dagger$, J. Kim ${ }^{2}$, F. J. Sánchez-Salcedo ${ }^{3}$, \\ J. Franco ${ }^{3}$ and L. Hernández-Cervantes ${ }^{3}$
}

${ }^{1}$ Dirección General de Servicios de Cómputo Académico, UNAM, 04510, Mexico City, Mexico email: alfredo@astrosu.unam.mx

${ }^{2}$ Korea Astronomy and Space Science Institute, 61-1, Hwaam-dong, Yuseong-gu, Daejeon, Republic of Korea 305-348

${ }^{3}$ Instituto de Astronomía , UNAM, 04510, Mexico City, Mexico

\begin{abstract}
We know that the galactic magnetic field possesses a random component in addition to the mean uniform component, with comparable strength of the two components. This random component is considered to play important roles in the evolution of the interstellar medium (ISM). In this work we present numerical simulations associated with the interaction of the supersonic flows located at high latitude in our Galaxy (High Velocity Clouds, HVC) with the magnetized galactic ISM in order to study the effect that produces a random magnetic field in the evolution of this objects.
\end{abstract}

Keywords. Magnetic fields - ISM: clouds - ISM: magnetic fields - ISM: structure

\section{Introduction}

Numerical simulations of the evolution of HVC collisions with the Milky Way have been performed for more than two decades by different authors. The details of resulting supersonic flows depend of on the model assumptions, and the intensity and initial configuration of the magnetic field. Santillán et al. (1999) made models that illustrate the effects of magnetic pressure, and differentiate them from those due to magnetic tension. The evolution of the interaction of a HVC with a magnetized interstellar medium, is studied by setting a random magnetic field that satisfies the divergence-free constraint $(\nabla . \mathbf{B}=0)$ at all times. To mimic the average galactic magnetic field, that is oriented parallel to the disk, the horizontal component dominates over the vertical component.

\section{Results}

We perform simulations of HVCs interacting with the interstellar medium without and with magnetic field, ISM-1 and ISM-2, respectively, using the MHD code ZEUS3D (Stone \& Norman 1992a,b). The ISM-models are plane parallel and have constant density and temperature, $n=1 \mathrm{~cm}^{-3}$ and $T=1000 \mathrm{~K}$. The galactic gas is initially at rest. For ISM-2 case the total intensity of the magnetic field is $2 \mu \mathrm{G}$. The enter position of the HVC is located $3 \mathrm{kpc}$ above from the midplane and has a velocity of $100 \mathrm{~km} / \mathrm{s}$. In the figure 1, the density is shown in logarithmic gray-scale plots and the magnetic field is indicated by arrows.

The early times evolution for both models display the same characteristics, e.g. the interaction between the $\mathrm{HVC}$ and the halo gas creates a strong galactic shock directed

$\dagger$ Present address: Unidad de Investigación en Cómputo Aplicado-DGSCA, UNAM, 04510, Mexico City, Mexico 

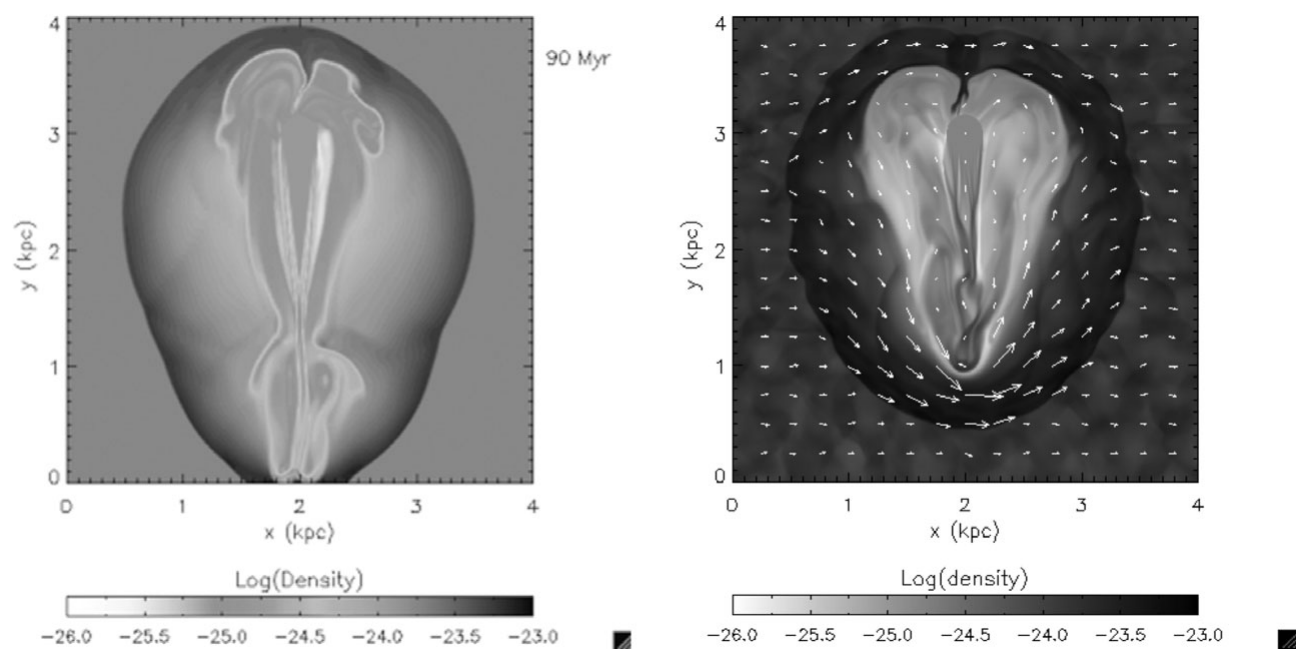

Figure 1. Evolution of HVC in two medium: without and with magnetic field. The figure shows the density (gray logarithmic scale) and the magnetic field indicated by arrows at 90 Myr.

downwards, along with reverse shock that penetrates into the cloud. The galactic shock tends to move radially away from the location of impact (Santillán et al. 1999). As time proceeds, the structure of the HVC changed slightly; in both models a large fraction of the original cloud mass remains locked up in the shocked layer, and a small amount of it re-expanded back into the rear wake and tail. However, in the HD-case, the clouds moves a greater distance than the MHD-case, due to the effect of the magnetic tension of the horizontal-component of random magnetic field. In the magnetic case, the HVC distorts and compresses the B-field lines during the evolution, increasing both the field pressure and the tension, and forming a magnetic barrier for the moving gas. Finally, as seen in figure 1, the late times structure produced by hydrodynamic simulations is completely different from that of its magnetic counterpart. In the case non-magnetic case, ISM-1, the interaction of $\mathrm{HVC}$ with ambient medium creates a thin structure and the size of perturbation region at $90 \mathrm{Myr}$ has grown to nearly $3 \mathrm{kpc}$. On the other hand, for the magnetic case, the evolution of the cloud at the same evolutionary time creates a thick structure of $\sim 2 \mathrm{kpc}$ and a great amount of gas collects in a magnetic valley formed by the interaction with the horizontal-component of the random magnetic field.

The numerical calculations were performed using UNAM's supercomputers.

\section{Acknowledgements}

This work has been partially supported from DGAPA-UNAM grant IN104306 and CONACyT proyect CB2006-60526.

\section{References}

Santillán A., Franco, J., Martos, M., \& Kim, J. 1999, ApJ 515, 657

Stone, J. M. \& Norman, M. L. 1992a, ApJS 80, 753

Stone, J. M. \& Norman, M. L. 1992b, ApJS 80, 791 